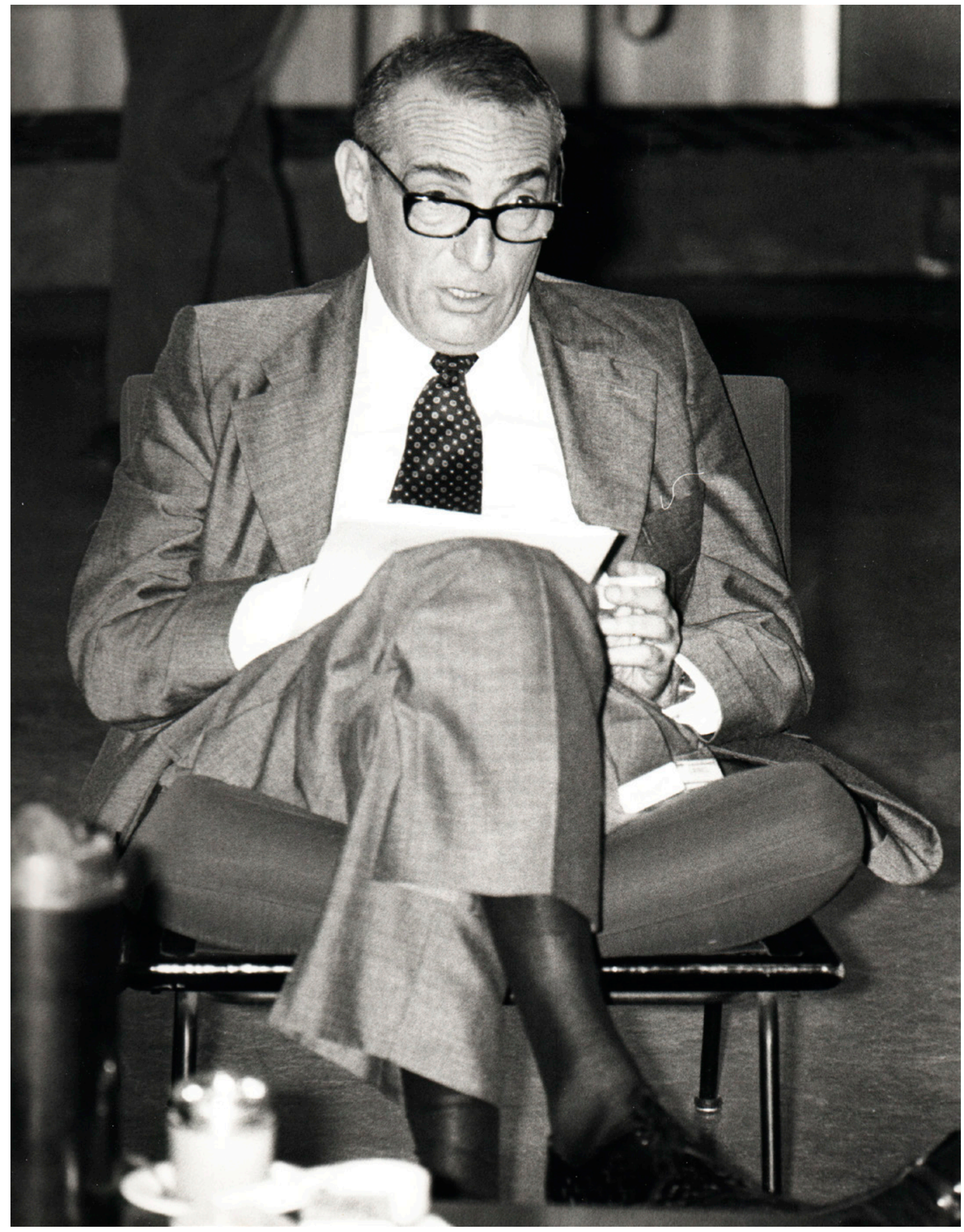

Camel Award Giovanni, sin datar. Archivo Herederos Vicente Aguilera Cerni. 


\title{
NUEVAS APORTACIONES A LA BIOGRAFÍA INTELECTUAL DE VICENTE AGUILERA CERNI. REFLEXIONES EN EL CENTENARIO DE SU NACIMIENTO
}

\author{
NEW CONTRIBUTIONS TO THE INTELLECTUAL \\ BIOGRAPHY OF VICENTE AGUILERA CERNI. \\ REFLECTIONS ON THE CENTENARY OF HIS BIRTH
}

Palabras clave

Abstract

Keywords
Lydia Frasquet Bellver Doctora en Historia del Arte

Resumen

Vicente Aguilera Cerni (1920-2005) es uno de los críticos e historiadores del arte españoles del siglo xx con mayor proyección internacional. La reciente investigación llevada a cabo sobre su biografía desvela una serie de datos que nos conduce a reflexionar sobre la visión panorámica de su trayectoria. Aguilera desarrolló una importante labor que debe ser reivindicada, puesto que practicó con firmeza una crítica y una promoción del arte comprometida con su época bajo sus particulares circunstancias. En el centenario de su nacimiento recordamos su figura como teórico del arte y creador del Museu de Vilafamés, uno de los más singulares de nuestro país.

Biografía intelectual, diplomacia cultural, crítica de arte, arte y política, arte durante el Franquismo, arte español contemporáneo.

Vicente Aguilera Cerni (1920-2005) is one of the most famous Spanish art critics and historians of the 20th century around the world. Recent research conducted on his biography has revealed some information that makes us reflect on the panoramic vision of his career. The importance of Aguilera's work should be duly recognized as he firmly conducted a form of critique and promotion of art that was committed to his time under his particular circumstances. On the centenary of his birth, we remember his figure as an art theoretician and founder of the Museum of Vilafamés, one of the most unique museums in our country.

Intellectual Biography, Cultural Diplomacy, Art Criticism, Art and Politics, Art during Franco's Regime, Contemporary Spanish Art. 
Vicente Aguilera Cerni es uno de los críticos e historiadores de arte españoles del siglo xx con mayor proyección internacional. Recientemente se ha publicado Vicente Aguilera Cerni y el arte español contemporáneo, monografía de la autora de este artículo, que se plantea como el primer acercamiento a su legado cultural y activista. La obra, resultado de una tesis doctoral dirigida por José Martín Martínez y codirigida por Juan Ángel Blasco Carrascosa, expone el papel diferente que Aguilera cumplió respecto a otras figuras del mismo momento histórico, al creer y practicar firmemente una crítica y una promoción del arte comprometidas con su época bajo sus particulares circunstancias. Igualmente, el estudio se hace eco de uno de sus mayores logros, la creación del llamado, en su momento, Museo Popular de Arte Contemporáneo de Villafamés y que ahora lleva su nombre. Un doble motivo: el del centenario de su nacimiento y el cincuentenario de la fundación del Museo, nos impulsan a escribir este texto.

A lo largo de mi investigación, el hecho de trabajar directamente con el archivo personal de Vicente Aguilera, custodiado por su hija Mercedes, ha supuesto una oportunidad para localizar las aportaciones más relevantes al conocimiento de la trayectoria del crítico. Tener acceso a este conjunto documental ha sido esencial para situar muchos de los episodios vitales de Aguilera. También se han encontrado cartas y documentos relacionados directamente con él en diversos archivos repartidos por la geografía española, del que destaca -por custodiar el informe sobre Aguilera del Ministerio de Información- el Archivo General de la Administración en Alcalá de Henares.

Junto a esta documentación novedosa, la dimensión de la figura del crítico valenciano se va precisando a través de una serie de entrevistas realizadas a los intelectuales $\mathrm{y}$ artistas que trabajaron o compartieron su tiempo con él. Las entrevistas a coetáneos son siempre una fuente muy valiosa para situar a las personas sobre las que se investiga. En este caso, los testimonios aportaron, enriquecieron y aclararon aspectos de la biografía de Aguilera y de su desarrollo profesional, al tiempo que han ayudado a comprobar cómo se recibió la obra de Aguilera en su momento. Se ha entrevistado a Salvador Aldana, Mercedes Aguilera, Doro Balaguer, Román de la Calle, Juan Ángel Blasco Carrascosa, Emèrit Bono, Víctor Fuentes Prósper, Manuel García García, Jacinta Gil, Beatriz Guttmann, Jesús Huguet, José Huguet Chanzá, Tomàs Llorens, Salvador Montesa, Luis Prades, Rosalía Sender, José Soler Vidal [Monjalés], José María Yturralde y Amparo Zaragozá. Todas estas personas nos han aportado datos muy valiosos sobre su figura y legado.

Vicente Aguilera Cerni nace en 1920, y es un joven inquieto que con 16 años frecuenta las filas de la izquierda estudiantil, y que, al estallar la Guerra Civil, se suma a ella como voluntario. A su regreso escribe y lee sin descanso. Según el Informe del Ministerio de Información y Turismo antes mencionado, mantiene su vinculación con el PCE clandestino y con organizaciones intervenidas por la policía, quedando finalmente desconectado del grupo principal.

Los inicios profesionales de Aguilera los situamos entre 1953 y 1958. Durante este periodo abandona la actividad filocomunista iniciada en su juventud. Es ahora cuando se vincula a una institución pública, lo que seguramente contribuye a este paréntesis. Su primer trabajo fue en el Servicio de Estudios Artísticos de la Institución Alfonso el Magnánimo de la Diputación de Valencia entre 1954 y 1959. Allí desarrolló tareas como investigador sobre arte en la Edad Media con el apoyo de Felipe María Garín Ortiz de Taranco, quien ostentaba cargos de responsabilidad en la ciudad bajo las instrucciones del régimen. En este ámbito de trabajo -y gracias a la correspondencia que existe entre ellos- ya se revela su voluntad de vincular sus primeros artículos sobre arte medieval con el notable hispanista de la Universidad de Harvard 


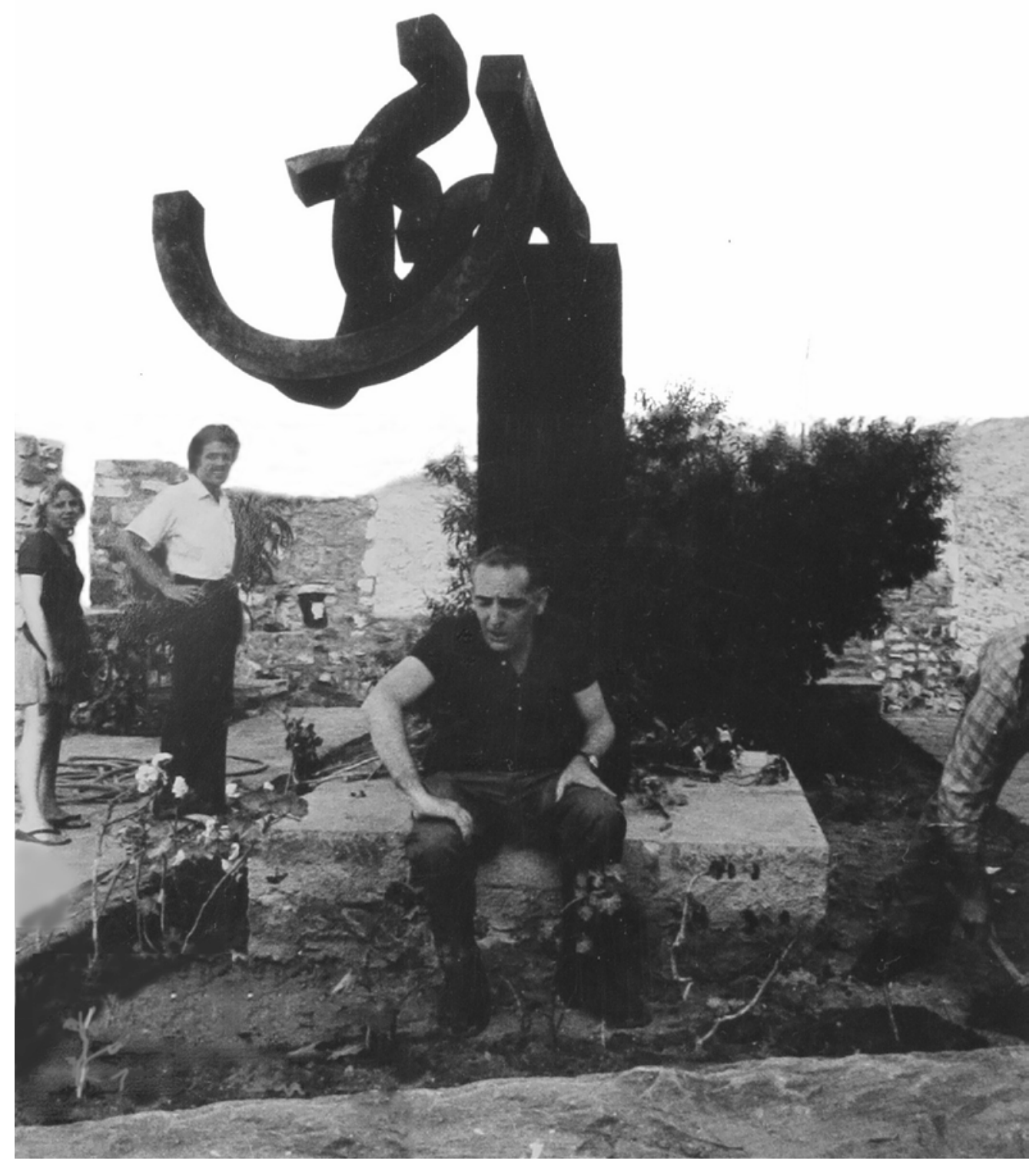

En primer plano, Vicente Aguilera Cerni en el patio del MACVAC de Vilafamés, junto a la escultura de Eduardo Chillida. Fotografía: Pepe Agost.

el profesor Chandler Rathfon Post. Esta faceta medievalista, del que fuera sin embargo uno de los grandes críticos de arte contemporáneo español, ha sido analizada por Juan Ángel Blasco Carrascosa (2016: 59-71), quien subraya además otros iniciales intereses literarios ahora ya bien conocidos.

Pronto comienza a publicar estudios sobre arte americano, y ha salido a la luz, gracias a la documentación de su archivo personal, que el servicio de información de los Estados Unidos respalda su carrera entre 1954 y 1959: le encargan conferencias para sus numerosos centros en España (las Casas Americanas), publica en sus revistas y medios de comunicación e incluso llega a formar parte de su equipo de dirección. En contrapartida, el servicio de información del gobierno americano se fija en Aguilera para 


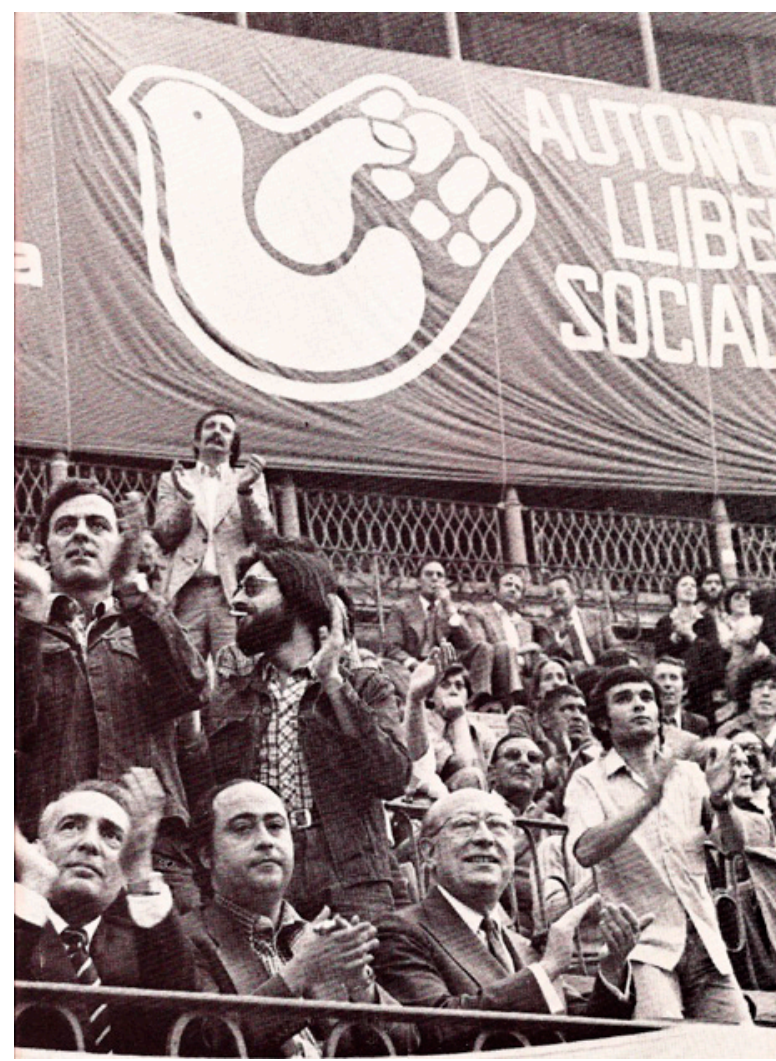

Aguilera junto a Sánchez Ayuso y Tierno Galván, 1977. Archivo Herederos Vicente Aguilera Cerni.

servir de altavoz de los valores americanos en territorio español.

En 1956 comienza la andadura el Grupo Parpalló con Aguilera como coordinador de la formación. Se han reunido numerosos testimonios orales de los artistas que explican y ratifican el papel que jugó Aguilera al ser el responsable de enfocar el grupo hacia la modernidad.

El periodo álgido de su carrera se desarrolla en los años siguientes, entre 1959 y 1964, en los que poco a poco deja ver su compromiso político con la izquierda. Un hecho muy relevante en su biografía es la recepción del premio de la crítica de la Bienal de Venecia en 1959; su carrera cobra en ese momento una proyección internacional. Tras su concesión, el régimen pasa a encargarle proyectos, como ser el comisario español de la III Bienal de Alejandría, entre otros. En consecuencia, Aguilera ha de escuchar la voz discrepante de los artistas. Es decir, que está inmerso en el debate de aceptar o no la promoción del arte contemporáneo que realiza el régimen.

En el ámbito editorial, dirige la revista Suma y sigue del arte contemporáneo entre 1962 y 1967, auténtica plataforma de intercambio internacional desde la que apoyó el arte normativo y seguidamente las nuevas formas de realismo social y crítico. Se considera un hito en un momento en el que se carecía de este tipo de publicaciones. Se afirma que es una de las revistas especializadas en artes visuales y arquitectura más destacadas del periodo del desarrollismo español (19621968). Su puesta en marcha está considerada como uno de los logros más destacables de Aguilera, y muchas de las personas a las que hemos entrevistado afirman que tuvo una repercusión muy relevante en la vida intelectual española de los años sesenta.

En 1965 Aguilera da forma al término Crónica de la Realidad, propugnando una pintura que aúna rasgos del pop con contenido social. Este término gozó de un éxito quizá inesperado, tanto que permanece actualmente en el uso bibliográfico más común.

Estos años, entre 1961 a 1965 y 1967, participa en los encuentros más relevantes de la crítica militante europea, los congresos de Rímini, dirigidos por Giulio Carlo Argan, y que tenían su plasmación en las Bienales de San Marino. Aguilera traslada a España las ideas allí discutidas (la alternativa al informalismo, la relación entre arte y ciencia) y anima a los artistas españoles a participar en estos cónclaves. El Ministerio del Interior tenía registrado su paso por estos encuentros. La correspondencia entre Aguilera y Argan nos revela el papel que desempeña en la creación de una sección nacional española de críticos dentro de un organismo internacional como AICA (Asociación Internacional de Críticos de Arte). Además, es Aguilera quien moviliza a los críticos españoles para unirse a esta plataforma y a defender el oficio. 


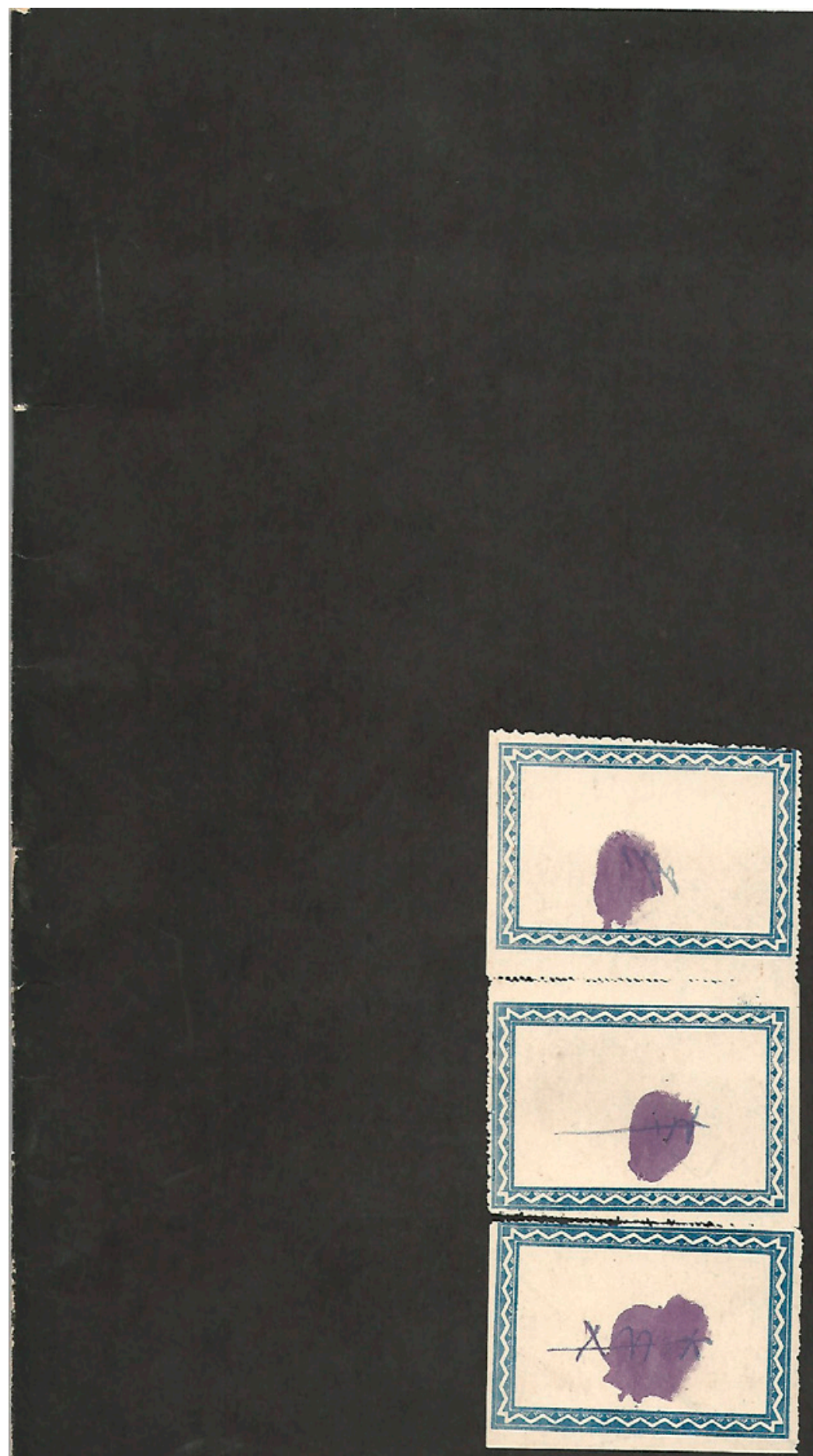

\section{suma y sigue del arte contemporáneo}

Monjalés, portada para Suma y sigue del arte contemporáneo, número 3, abril-mayo-junio de 1963. Fuente: Centro Internacional de Documentación Artística de Vilafamés. 
Será Aguilera el encargado de seleccionar a determinados artistas españoles para participar en la mítica exposición celebrada en Italia titulada «España Libre» (1964-1965), en la que se mostraba arte al margen del control del régimen. Abiertamente se le considera crítico militante, frente a la postura más ambigua de solo tres años atrás, como comisario del envío oficial español de la Bienal de Alejandría.

Entre 1965 y 1976 desarrolla una serie de proyectos en los que ya se muestra contrario al régimen. Por ejemplo, en 1965 participa en el Congreso Mundial por la Paz en Helsinki, organizado por el departamento internacional del Comité Central del Partido Comunista soviético. El dosier policial del Ministerio de Información califica su trayectoria como la de persona desafecta al régimen. En pocos años ha pasado de amigo del imperialismo americano a compañero de viaje de los comunistas.

En el plano profesional, publica en 1966 su obra posiblemente más citada: Panorama del nuevo arte español, en la que aborda el arte desde 1940 a 1966. Publica ese mismo año Ortega y D'Ors en la cultura artística española, y poco después Iniciación al arte español de la postguerra, además de varios libros que recopilan una selección de sus artículos, entre otras muchas publicaciones y textos para catálogos. Un dato poco conocido que hemos sacado a luz es que el Ministerio de Educación y Ciencia le encargó al menos seis guiones para documentales de arte, entre los años 1966 y 1971.

Pasan los años, sufre el desencanto tras la Primavera de Praga, y Aguilera decide vincularse con el proyecto del profesor Tierno Galván. Su compromiso político le lleva a desempeñar el cargo de presidente del Partido Socialista Popular-pV entre 1975 y 1978.

En estos años pone en marcha varias iniciativas innovadoras, como la denominada Antes del arte, que se desarrolló entre 1968 y 1969 y en la que se exploraron las relaciones entre el arte $y$ la ciencia. Reunió a los artistas Joaquín Micha- vila, Eduardo Sanz, Eusebio Sempere, Francisco Sobrino, Ramón de Soto, Jordi Teixidor y José María Yturralde. Este último destaca cómo esta experiencia marcó la trayectoria de todos sus componentes.

Emprende la aventura de fundar un museo, el primer centro de arte contemporáneo de la Comunidad Valenciana, que abre al público en Vilafamés (Castellón) en 1972. Aguilera lo concibe como un museo popular, democrático y renovable frente a la idea de museo elitista, que nos habla de su voluntad de cambio y de traer aires de modernidad. Nuestro biografiado configura este museo de una manera inusual, y es que solicita las obras a los autores en régimen de cesión o de donación, y esa fórmula se considera pionera en nuestro territorio. La colección así formada tiene la capacidad de cambiar y ser dinámica. En cualquier caso, hay que recordar que la colección se configura como una selección realizada por Aguilera. Además ostenta la característica de ser una de las primeras instituciones museísticas dedicadas al arte contemporáneo en España.

En 1972 se inaugura en Valencia la Galería Punto. El matrimonio que la gestiona, formado por Miguel Agrait y Amparo Zaragoza, buscan el asesoramiento de Aguilera desde el inicio de su andadura, y trabajará con ellos durante los quince años siguientes. La labor de Aguilera en Punto posibilita que pueda introducir a los artistas de sus líneas de pensamiento y ayuda a crear un espacio de vanguardia.

Poco después, en 1975, se produce un punto de inflexión con dos sucesos que dejan a Aguilera en una situación profesionalmente comprometida: el encargo -frustrado- de la muestra española en la Bienal de Venecia de 1976, que finalmente realizará otro equipo de especialistas capitaneados por Tomàs Llorens y Valeriano Bozal. La correspondencia entre Aguilera y Renau informa de los intentos -sin éxito- de Aguilera por movilizar un manifiesto de protesta.

Otro suceso comprometido acaece en torno a la exposición promovida desde el Ayun- 
tamiento de Valencia titulada «75 años de pintura valenciana». Aguilera forma parte de la comisión organizadora, pero no encuentra apoyo en los artistas que se alzan en contra por considerarla una propuesta todavía vinculada al régimen. Nos encontramos así ante un Aguilera controvertido, que vive dos realidades opuestas: es reconocido y admirado en Italia, cuestionado en su ciudad. Paradójicamente, en 1976 la prestigiosa revista Bolaffiarte de Turín realiza una encuesta para elegir al hombre del año entre las personalidades del mundo del arte. Las respuestas son unánimes, y Aguilera es proclamado el ganador. A la vez, los artistas y críticos de su ciudad se han alzado en contra por no representar la opción para ellos más coherente para el momento histórico, la anhelada ruptura con el franquismo. Aguilera y la política del PCE eran más partidarios de la «reconciliación nacional».

Como consecuencia de estos sucesos pierde la posición de indiscutible líder cultural que ostentó durante tantos años, pero está activo prácticamente hasta el año 2005. Dirige la Historia General del arte valenciano en 6 volúmenes (1986), una obra que todavía hoy no ha sido superada en su ámbito. Entre 1979 y 2003 aborda la dirección de la tercera revista de su trayectoria, Cimal, todo un referente entre las publicaciones españolas de cultura visual contemporánea. Desde esta plataforma Aguilera apuesta de nuevo por ofrecer información crítica sobre la modernidad, planteando tanto textos de reflexión teórica como de información y crítica artística, sin perder de vista el horizonte europeo. Aguilera fue el encargado de reunir las muchas voces críticas que hablasen de manera rigurosa de la creatividad de su momento.

También en estos años se funda la Asociación Valenciana de Críticos de Arte, cuya presidencia ejerció entre 1980 y 1987, testimoniando su voluntad de respaldar el oficio. Este mismo año se celebra, por tanto el cuadragésimo aniversario de su puesta en marcha.
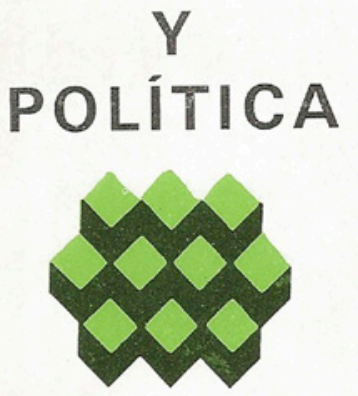

ALIANZA POPULAR

PARTIT COMUNISTA DEL P.V.

PARTIDO DEMÓCRATA LIBERAL

PARTIDO LABORISTA

PARTIDO POPULAR

PARTIDO SOCIALISTA OBRERO ESPAÑOL PARTIDO SOCIALISTA POPULAR PARTIT SOCIALISTA DEL P.V. UNIÓ DEMOCRÁTICA DEL P.V.

ENCUENTROS DE VILLAFAMĖS

Cultura y política, 1977. Fuente: Centro Internacional de Documentación Artística de Vilafamés.

Creemos relevante también poder datar su compromiso con las filas del socialismo, ya que en 1981 Ciprià Císcar (conseller de Cultura del gobierno socialista) lo nombra asesor para el ámbito cultural. Hasta el último de sus días, Aguilera mantuvo su afiliación al psoe. Se le requiere para un organismo consultivo denominado Consell Valencià de Cultura (CVC) desde 1985, ejerciendo su presidencia entre 1994 y 1996. Entre las tareas que desempeñó podemos destacar la recuperación de piezas de autores valencianos enviadas al Pabellón español de la Exposición Internacional de París de 1937. En opinión del 
SEMANA DE CULTURA NORTEAMERICANA

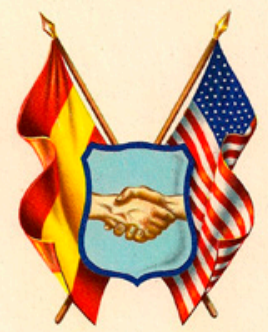

ATENEO MERCANTIL DE VALENCIA MAYO 195

Díptico de la Semana de Cultura Norteamericana en el Ateneo Mercantil de Valencia, 1954. Archivo Herederos Vicente Aguilera Cerni.

actual secretario, Jesús Huguet, la aportación más señalada que realizó Aguilera desde la presidencia fue la producción editorial, concretamente la introducción de las artes plásticas en las publicaciones. Al mismo tiempo, la prensa de su archivo deja ver cómo queda relegado de los proyectos que las nuevas estructuras culturales están poniendo en marcha.

En los últimos años de su vida recibe galardones y reconocimientos institucionales, pero la generación de la Transición, tanto críticos como artistas, no ve en él la figura del intelectual. Sin embargo, nos deja vigente su legado, en el que plasmó la firme convicción de que el arte y la cultura, desde la libertad, eran los pilares sobre los que construir la mejor sociedad posible.

Estas nuevas aportaciones nos permiten arrojar varias miradas sobre su trayectoria vital.
Tuvo un posicionamiento político muy confuso, propio de un contexto de postguerra y de un régimen represivo, manteniendo una posición poco definida. Como se ha señalado, su carrera no es una línea unívoca y a lo largo de su trayectoria ejecuta determinadas acciones con y contra el régimen, desarrolladas prácticamente en los mismos periodos temporales.

En la trayectoria de Aguilera existieron ocasiones en las que pudo pronunciarse y denunciar al régimen. En otras, en cambio, acepta encargos directos. Muy probablemente se trataba de una actitud precavida, y prefirió pronunciarse en ámbitos que él contaba como seguros, como los encuentros de la AICA, donde se permitía hablar claro sobre la situación española y demostrar su oposición a la dictadura. En este sentido, Aguilera sería uno de los personajes atrapados en esa paradoja que se dio en el arte español contemporáneo, al ser utilizado y dejarse utilizar por el régimen para su promoción en el exterior.

Esta situación poco definida tendrá sus consecuencias ya que se verá rechazado por la siguiente generación de críticos y artistas, mientras había sido considerado desafecto por el régimen franquista. Podemos afirmarlo gracias al acceso brindado a la documentación personal de Aguilera, así como a su informe ministerial.

Con mucha probabilidad, Aguilera se implicó lo que pudo en la denuncia de la situación española, y se arriesgó cuando lo consideró oportuno, aunque nunca llegó a tomar posiciones que le llevasen hasta la cárcel, como lo hizo su coetáneo José María Moreno Galván. Tal vez por eso, a los ojos de los protagonistas de la siguiente generación -Tomàs Llorens, Valeriano Bozal, por ejemplo- su posición no fue clara y su postura poco comprometida.

Si bien sus escritos no sufrieron el ataque de la censura, sí en cambio era observado por la dictadura debido a su relación e implicación con determinadas instituciones y organizacio- 
nes, así como por su participación en cónclaves muy relevantes.

Los documentos guardados en su archivo personal revelan que entre 1954 y 1959 Aguilera formó parte de la red cultural norteamericana que se había instaurado en España en 1945. El trabajo que el crítico realiza dentro de esta plataforma cultural tiene tal peso (publica artículos, libros, imparte charlas por muchos de sus centros en España) que modifica la lectura de su trayectoria. Hasta ahora creíamos simplemente que había sido el pionero en escribir sobre historia del arte norteamericano en España, y en realidad va más allá: fue considerado por las instituciones vinculadas al Servicio de Información Americano (UsIs) como una persona lo suficientemente influyente, capaz de difundir los valores de la vida y la cultura norteamericana a través de su trabajo. Por este motivo, se impulsó su carrera, ofreciéndole sus medios y plataformas culturales para publicar y seguir investigando sobre cultura norteamericana.

Aguilera contribuye a abrir la brecha de aislamiento cultural que había impuesto la dura postguerra en toda la Península. Esta apertura la lleva a cabo desde varios frentes, desde el ámbito de la gestión de exposiciones y colectivos, y desde la activación de la publicación de escritos.

La posición profesional de Aguilera -no es docente en la escuela de Bellas Artes ni ostenta ningún puesto dentro de la organización franquista- le permite mantener la independencia, que le posibilita activar determinados colectivos o grupos. Estos colectivos surgen porque dentro de la escuela, sometida por el régimen, no es posible mantener contactos con otros centros.

En un contexto yermo, Aguilera realiza el papel, tan necesario, de servir de mediador entre los artistas y las instituciones. Él es el facilitador de esas primeras exposiciones en centros y organizaciones (como el Ateneo, etcétera), tan necesarias para el desarrollo por parte de los artistas de sus obras. También es el impulsor de esas primeras publicaciones con voluntad de cambio, y de generar un espacio social nuevo para la creatividad en la ciudad de postguerra. Ese papel lo desempeña con el Grupo Parpalló, con el realismo social y con Antes del arte. Este rol lo ejerce pidiendo desde sus escritos a los artistas que realicen su trabajo con una actitud responsable hacia la sociedad en la que viven.

Otro de los roles que desempeñó Aguilera en la década de los sesenta y principios de los setenta fue el de ejercer un papel patriarcal o de paraguas bajo el que adoptar artistas, demostrando facilidad para aglutinarlos bajo denominadores e impulsarlos dentro de una tendencia.

Aguilera realiza un trabajo de compilación, enumerando quién participa en este momento en la historia del arte español; esa relación es un trabajo necesario, ya que constituye el primer escalón para que la siguiente generación dé un paso más allá y pueda teorizar sobre esa primera larga lista de nombres, hechos y movimientos. Juega por tanto un papel esencial, al realizar lo que se considera la primera tarea para avanzar en esta disciplina y lo ejerció con buen criterio de calidad.

Esta voluntad integradora la plasmó en varias de sus obras, y muy notablemente en Panorama del nuevo arte español (1966), en el que ofreció un exhaustivo estado de la cuestión en un momento en el que apenas existían publicaciones sobre creatividad contemporánea, y ninguna con esa ambición compiladora. Con el transcurrir de los años, su actitud inclusiva muy posiblemente también le pasará factura.

A lo largo de su trayectoria, Aguilera realiza una labor en ambos sentidos: exportando el arte español contemporáneo fuera de las fronteras de la Península y ayudando a importar a España las ideas estéticas de 
modernidad. Su trabajo de difusión del arte español hacia el exterior, sobre todo hacia Italia, se plasma en la selección de artistas y obras, posibilitando que determinados creadores españoles fueran visibles en los encuentros del momento en los años sesenta: selecciona obras para la Bienal de Alejandría, para la muestra «España Libre», para la relevante exposición de la galería Marlborough de Londres, entre otras muchas. El hecho de sumarse a la AICA desvela su voluntad de ser portavoz y plataforma de los artistas en un escenario internacional. Aguilera, a lo largo de su carrera, hace de puente entre Valencia y el panorama internacional.

Al mismo tiempo, ejerce con seguridad el movimiento inverso, trasladando la influencia de lo que se estaba creando en el exterior del país, difundiendo y escribiendo sobre arte norteamericano al inicio de su carrera, introduciendo teorías a través de sus escritos, sus charlas, reseñando los libros que se publicaban en Francia e Italia, incluso promoviendo su traducción al castellano.

Una última reflexión: innegablemente es Aguilera quien ayuda a activar la vida artística valenciana de postguerra, quien contribuye a generar un espacio de desarrollo y de debate a través del respaldo a grupos y de posibilitar que se celebren exposiciones en instituciones. En el campo de la crítica, es quien le proporciona entidad, al crear revistas en las que otros críticos pueden publicar, pero va más allá y convierte estas revistas en plataformas desde donde difundir en España las voces críticas de la modernidad, contando con textos de colaboradores extranjeros. Contribuye, a través de sus escritos, a que la crítica tenga una voz de calidad. Genera un espacio de colaboración para los artistas en el Museo de Vilafamés. Colabora a impulsar una galería de arte en la ciudad; redacta un diccionario y una Historia del arte valenciano, escribe en la prensa diaria, crea la Asociación Valenciana de Críticos de Arte, en definitiva, pone en marcha proyectos que en su momento fueron novedosos y necesarios para el desarrollo tanto de las carreras de los artistas como de los críticos que trabajaron con él. En conclusión, podemos afirmar que la labor emprendida por Aguilera genera un espacio -que no existía en el entramado social español- para el arte contemporáneo, para la historia del arte, para la historia de la crítica y para la historia del pensamiento.

\section{REFERENCIAS Y BIBLIOGRAFÍA}

Aguilera Cerni, Vicente (1966) Panorama del nuevo arte español, Madrid: Guadarrama.

- (1966) Ortega y D’Ors en la cultura artística española, Madrid: Ciencia Nueva.

- (1970) Iniciación al arte español de la postguerra, Barcelona: Península.

- (1969) El arte impugnado, Madrid: Cuadernos para el diálogo.

- (1973) Posibilidad e imposibilidad del arte. Comentarios en el tiempo, Valencia: Fernando Torres.

Archivo Herederos de Vicente Aguilera Cerni, custodiado por su hija Mercedes Aguilera Ingunza.

Blasco Carrascosa, Juan Ángel (2016) «Algo más sobre la vocación medievalista de Vicente Aguilera Cerni», Diferents. Revista de museus, 1: 59-71.

Frasquet Bellver, Lydia (2020) Vicente Aguilera Cerni y el arte español contemporáneo, Valencia: Institució Alfons el Magnànim.

Informe sobre Vicente Aguilera Cerni (1967) Ministerio de Información y Turismo. Archivo General de la Administración, Alcalá de Henares.

Firma invitada BIBLID [2530-1330 (2020): 6-17] 


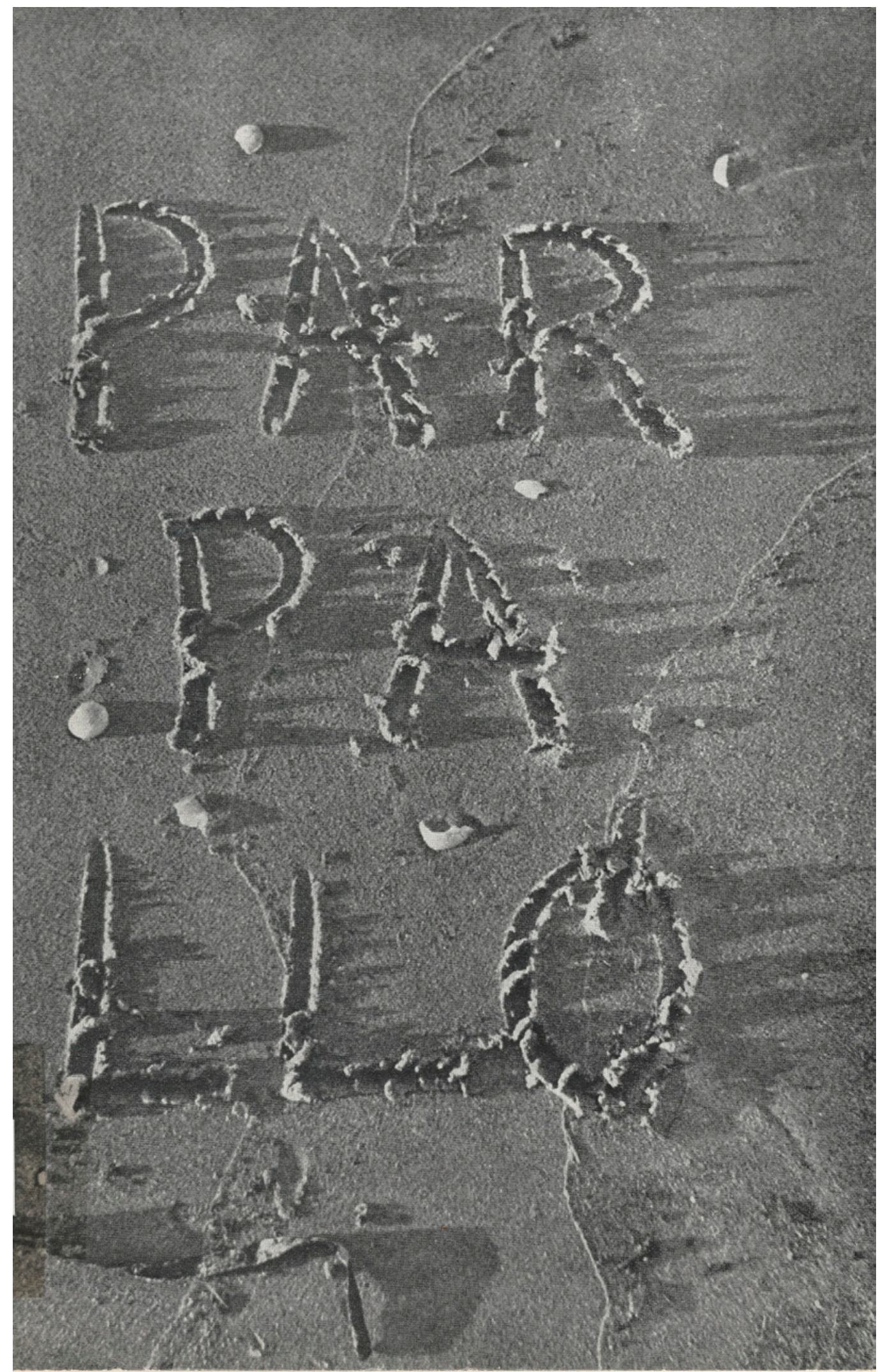

Portada del catálogo del Grupo Parpalló. Sala Gaspar de Barcelona, 1959. Fuente: Centro Internacional de Documentación Artística de Vilafamés. 